Florence Olivier

\title{
Trois intraduisibles (et traduits) romans régionalistes latino-américains
}

\author{
La vorágine, Don Segundo Sombra et Doña Bárbara
}

Pourrions-nous ou devrions-nous penser toute la littérature comme un intraduisible corps verbal ? Pourquoi pas, si l'on s'en tient aux réflexions théoriques, qui ne portent pas sur l'intraduisible mais sur les " intraduisibles », qu'a conduites dernièrement Barbara Cassin tandis que s'élaborait sous sa direction Le vocabulaire européen des philosophies. Dictionnaire des intraduisibles (2004). Pour définir les « intraduisibles » la philosophe emprunte à Derrida la formulation : "ce qu'on ne cesse pas de (ne pas) traduire » (Cassin 2016: 54), et s'appuie sur sa formation d'helléniste et sur sa posture de sophiste pour faire l'éloge de la traduction comme pratique permettant de penser la dissociation entre le logos et le langage. En effet, souligne-t-elle, nous pensons selon la langue dont nous usons pour ce faire, nous philosophons en (différentes) langues. Convoquant dans le plein texte tout ce qui, dans les traductions de textes philosophiques, se voit d'ordinaire indiqué entre parenthèses ou relégué dans des notes de bas de page, l'entreprise du Vocabulaire européen des philosophies met ainsi en lumière ces différences terminologiques qui trahissent des différences conceptuelles. Apparaît alors ce qui résiste à l'illusion d'une exacte équivalence terminologique d'une langue à une autre et qui, par là, questionne la prétention des concepts à l'universalité.

Ce qui peut nous intéresser, de cette machine à questionner l'universel ou à le « compliquer », c'est qu'elle manie la philologie en cheval de Troie pour pénétrer dans la Cité Philosophique. Très concrètement, elle fait appel à l'introduction de Wilhelm Von Humboldt à sa version allemande de l'Agamemnon d'Eschyle, parue en 1816. Jugeant cette œuvre intraduisible tout en la traduisant, le philologue et traducteur allemand tire des réflexions générales de son travail. Intraduisible, cette œuvre l'est à ses yeux à plusieurs titres : du fait, nécessairement, de sa grande originalité et de son intensité esthétique, qui lui ont donné un tel rôle fondateur dans la culture grecque ancienne que ces qualités sont difficilement transposables dans une autre culture et à une autre époque ; mais intraduisible, elle l'est aussi du fait de sa singularité, du fait, tout simplement, qu'elle est unique. Et Humboldt de commenter l'obscurité, à la première

Florence Olivier, CERC, Sorbonne Nouvelle Paris 3

○ Open Access. (C) 2022 Florence Olivier, published by De Gruyter. (cc) BY-NC-ND This work is licensed under the Creative Commons Attribution-NonCommercial-NoDerivatives 4.0 International License.

https://doi.org/10.1515/9783110707557-005 
lecture, de l'œuvre d'Eschyle, qui omet les « conjonctions intermédiaires » pour aligner les uns derrière les autres « des pensées, des images, des souvenirs et des pressentiments, issus d'une âme profondément remuée » (Humboldt, cité dans Cassin 2016 : 185). Barbara Cassin interprète bien évidemment cette remarque en rapportant le propos du philologue à la qualité véloce et synthétique de la tragédie d'Eschyle, c'est-à-dire à son rythme. Elle souligne qu'Humboldt signale tout le soin qu'il a mis à chercher une forme métrique juste et épurée à sa version allemande du texte grec. Humboldt entend la traduction d'un tel texte intraduisible comme une tâche inachevée et, en dernier recours, collective, dont les étapes consisteraient dans les successives versions traduites auxquelles l'œuvre aura donné ou donnera lieu, comme s'il s'agissait d'autant d'approches ou de gages au fil d'une cour amoureuse. Ainsi donc, l'intraduisible corps verbal du texte, sa matérialité rythmique et sonore, ne saurait se traduire, pour reprendre les termes du philologue, qu'en partant d'un « amour simple et sans prétention pour l'original » (Humboldt cité dans Cassin 2016 : 187), un amour dont le degré de fidélité trouve ses limites dans la discrétion avec laquelle se manifeste dans la version traduite la présence, non pas de l'étrangeté, mais de l'[élément] étranger. Au discret lecteur d'en juger, se fie Humboldt. Ce lecteur, lui-même érudit ou philologue, devient ipso facto le tiers entre l'auteur et le traducteur, l'autorité qui valide telle ou telle traduction de l'œuvre originale. Il ne s'y trompera pas s'il perçoit qu'elle n'est ni trop assujettie à la lettre de l'original ni dédaigneuse des effets que produit cette dernière.

Selon Derrida, que cite Barbara Cassin dans son Éloge de la traduction (2016), le corps verbal d'une langue serait ce qui ne saurait se transposer d'une langue à une autre, ce que la traduction délaisse ou dont elle fait abstraction, l'énergie spécifique de la traduction consistant dans ce geste même. Commentant ce propos, la philosophe ajoute :

Le corps des langues est ce avec quoi, par excellence, les traducteurs dits littéraires, et au premier chef les poètes-traducteurs et les traducteurs-poètes, sont confrontés, de l'intraduisible au singulier mais avec un $i$ minuscule, le signifiant en ses sonorités, rythmes, langues telles qu'on les expérimente, telles qu'elles existent (Cassin $2016: 69$ ).

Délimitant le champ de la philosophie de celui de la littérature tout en soulignant leur contigüité, tout particulièrement dans les traditions de langue italienne, espagnole et portugaise, Barbara Cassin cherche au premier un territoire plus ouvert. C'est à travers la différence qu'elle établit entre « l'Intraduisible » avec un $i$ majuscule, superstition tant de la traduction que de la philosophie adepte de la Vérité, et l'intraduisible avec un $i$ minuscule qui germe et se pluralise dans le Dictionnaire des intraduisibles, qu'elle revendique, face à la supposée unicité universelle du concept, la diversité conceptuelle liée à la diversité des langues. Ainsi, 
au pluriel, les « intraduisibles » des philosophies européennes sont des termes que le Vocabulaire assortit de commentaires et de leurs habituelles ou éventuelles traductions, sans qu'aucune d'entre elles ne soit désignée comme exacte et définitive. Cassin qualifie donc le Dictionnaire de « pluraliste et comparatif, en un geste sans clôture, beaucoup plus borgésien ou oulipien que destinal et heideggérien » (Cassin 2016 : 54).

Une distance considérable sépare les « intraduisibles », vocables et noyaux sémantico-conceptuels, de " l'intraduisible » comme corps verbal de telle ou telle langue, manié à des fins esthétiques dans un texte littéraire. Les « intraduisibles » des philosophies européennes, comptabilisés, ont été sélectionnés pour élaborer un vocabulaire comparatif. "L'intraduisible ", dès lors qu'il s'agit de traduire une œuvre littéraire, relève du signifiant situé dans l'horizon de la fonction esthétique du langage. Retenons néanmoins l'idée que tant les « intraduisibles » que " l'intraduisible » seraient ce qui ne cesse pas de (ne pas) se traduire, motivant un incessant travail de traduction, collectif en fin de compte, et par là, apparenté à la tâche de la recherche. De ce point de vue, qu'il s'agisse des « intraduisibles » terminologiques ou de " l'intraduisible » artistique, le travail du traducteur doit être pensé comme la quête non pas de la meilleure traduction, ultime et définitive, mais de la meilleure traduction en vue de tel ou tel effet de réception. Dans l'univers de la traduction littéraire, même lorsqu'il ne s'agit pas de retraduire une œuvre mais de la traduire pour la première fois, la tâche du traducteur, certes individuelle mais inscrite dans le processus collectif, diachronique et synchronique, de la traduction, pourrait être comparée à celle d'un chercheur en littérature ou d'un auteur se situant dans la dynamique entre tradition et création.

Mais laissons là, momentanément, la stimulante perspective globale de l'intraduisible corps verbal de la langue tel que le manie tel ou tel texte littéraire. Arrêtons-nous sur une seule de ses composantes, la plus inerte à première vue, le lexique, et rappelons-nous comment, dans une langue une et diverse telle que l'espagnol, certains termes locaux ou régionaux ont pu se voir valorisés littérairement comme intraduisibles. En l'occurrence, loin d'être des termes désignant des concepts prétendant à l'universalité, il s'agit de realia et ce statut leur donne un prix singulier : objets de collection, ils semblent aussi précieux que les plantes ou les animaux que, souvent, ils désignent. La revendication de tels termes, appréhendés depuis une norme linguistique nationale ou une norme qui assimilait le langage littéraire à un style " noble » ou « savant » dont on supposait qu'il transcendait les singularités des parlers nationaux de l'espagnol, fait partie de l'histoire littéraire hispano-américaine. Les canoniques romans dits « de la terre » qui furent publiés dans les années vingt du siècle dernier en Colombie, en Argentine et au Venezuela firent en effet le pari de donner droit de cité à un 
lexique - et à une syntaxe de l'oral - censément autochtones, qui caractérisent le parler d'une région dont l'identité naturelle et culturelle se voit explorée et souvent célébrée, au point qu'elle est érigée en emblème de l'esprit national. Trois titres constituent d'ordinaire le corpus exemplaire ${ }^{1}$ de l'ensemble de cette production dans les histoires de la littérature hispano-américaine : La vorágine (1924), roman des plaines orientales et de la forêt amazonienne du Colombien José Eustasio Rivera ; Don Segundo Sombra (1926), roman de la pampa de l'Argentin Ricardo Güiraldes ; et Doña Bárbara (1929), roman des plaines du Vénézuélien Rómulo Gallegos. Le geste qui consistait à transformer en territoires littéraires les plaines colombiennes ou vénézuéliennes, la forêt amazonienne, la pampa de la province de Buenos Aires revenait alors à poursuivre symboliquement la tâche civilisatrice et hautement politique d'une intégration nationale ou à alerter sur l'urgente nécessité de la mener à bien. Ces régions en viennent ainsi à apparaître dans les romans comme la terre promise de la nation, tout à la fois réserve naturelle et lieu d'un patrimoine culturel populaire. Comme s'il s'agissait de la part infantile ou juvénile, voire sauvage, du territoire et de la nation, demeurée dans l'attente d'une éducation à même de canaliser les qualités morales, virtuelles ou confirmées, de ses habitants.

À l'image de pionniers pénétrant dans une région de " frontière », les romanciers prétendaient, non sans audace lyrique, introduire ainsi un dialogisme entre parler populaire régional et langue savante nationale ; ils s'efforçaient de présenter une vivante pratique locale de l'oralité à la langue d'écriture nationale et internationale. Concrètement, l'intégration du parler populaire régional au récit romanesque se voit maniée dans les dialogues que tiennent les personnages locaux. Dans un souci de sauvegarde folkloriste de la poésie populaire, sont de surcroît transcrites des strophes ou des couplets dans d'occasionnelles scènes où rivalisent d'adresse les llaneros vénézuéliens ou les danseurs et les chanteurs d'un gato ou quadrille de la pampa. Cependant, la valorisation esthétique du parler et de la culture régionale, leur migration et leur installation dans la langue écrite littéraire, n’ont pas toujours été dissociées, par les auteurs et/ou leurs éditeurs, d'une nécessité de leur octroyer un sauf-conduit linguistique. Si les romanciers n'étaient guère inquiets, semblerait-il, de voir leurs lecteurs aux prises avec la syntaxe de l'oral, Rivera et Gallegos se crurent en revanche dans l'obligation de fournir la traduction dans un castillan censément commun des termes locaux, bien évidemment perçus comme étrangers ou forains à l'égard d'un

1 Et ce, dès leur première réception. Voir à ce propos l'article que publie en 1936 le Cubain Juan Marinello : «Tres novelas ejemplares (Don Segundo Sombra, Doña Bárbara y La vorágine) ». In : Bimestre Cubano, n³8, La Habana, 1936, pp. 234-249. 
centre symbolique, d'une capitale ou d'une métropole de la langue écrite. Ainsi, le sens des termes locaux ou simplement populaires, présents dans le récit romanesque et, dans le cas de La vorágine, placés entre guillemets ou notés en italique, se voit éclairci par des périphrases définitoires ou traduit par d'approximatifs synonymes dans un glossaire final.

Don Segundo Sombra fait exception, puisqu'en 1926 la première édition du roman chez l'avant-gardiste maison Proa de Buenos Aires ne comprenait aucun glossaire. Les éditions argentines qui suivirent en furent également dépourvues. Si la première édition, colombienne, de La vorágine (Bogota, Cromos, Luis Tamayo y Cía, 1924) ne proposait pas de glossaire, à la différence de la première édition, espagnole, de Doña Bárbara (Barcelone, Araluce, 1929), qui visait une éventuelle distribution internationale dans les pays de langue espagnole, il serait presque hasardeux d'attribuer cette divergence au milieu littéraire de leurs respectifs pays de publication, national ou péninsulaire. En effet, dès la troisième édition de son roman (Bogotá, Minerva, 1926), tout aussi colombienne que les deux premières, José Eustasio Rivera ajouta au récit un « Vocabulaire », de fait relativement bref, qu'il amplifia assez logiquement deux ans plus tard pour une édition new-yorkaise en castillan.

La fortune et l'évolution de ces glossaires originaux dans les éditions successives des romans se sont vues assujetties à des stratégies éditoriales redevables au pays et à l'époque de la publication, de même qu'au type de collection dans lesquelles paraissaient les romans. Pour des raisons moins divergentes qu'il n’y paraîtrait, ces glossaires ont été inclus aussi bien dans les éditions de collections populaires réalisées en Espagne que dans les éditions critiques des trois romans dans des collections aussi prestigieuses qu' « Archivos ALLCA-Unesco » ou "Letras Hispánicas » de la maison madrilène Cátedra. Hors d'Argentine, l'exception que constituait l'absence de glossaire dans la première édition de Don Segundo Sombra, heureux choix aux yeux du lecteur actuel, ne s'est pas maintenue, loin s'en faut, au long de l'histoire éditoriale du roman. Si la tendance qui a prévalu a été de conserver ces glossaires et, bien souvent, de les étendre à des fins scientifiques, l'on peut également observer un récent et relatif changement de paradigme dans l'appréhension académique de la norme lexico-linguistique d'un espagnol général censé être le garant de la lisibilité des œuvres dans tous les pays de l'univers hispanique. Ainsi, pour l'édition critique de Doña Bárbara qu'il élabore pour Cátedra en 1997, le Vénézuélien Domingo Miliani, spécialiste reconnu de l'œuvre de Gallegos, décide d'éliminer le glossaire original, non sans le remplacer par des notes de bas de page, invoquant la nécessité d'une plus grande précision lexicographique : 
El Vocabulario, al final del libro, incluido en las ediciones anteriores de la novela, fue sustituido por una secuencia de notas lexicográficas a pie de página. Ellas explican los americanismos [en este caso venezonalismos] en el contexto de la obra. Cuando los lexemas son explicados por Gallegos en la novela, se obvia su descripción en notas (Miliani dans Gallegos 1997 : 89).

En qualifiant de vénézualismes les termes de la région des Llanos, Domingo Miliani déplace partiellement, au bénéfice d'un public lecteur pan-hispanique, le critère qui semblait initialement régir le " Vocabulaire » : il le fait passer de l'échelle régionale/nationale à l'échelle nationale/internationale. Pour l'édition critique de La vorágine dans la même collection de Cátedra, la Colombiano-catalane Montserrat Ordóñez propose pour sa part une appréciation contemporaine du geste lexical de l'auteur, soulignant le changement de sensibilité et de critères à l'égard du caractère paradoxalement forain du lexique régional :

En primer lugar, se suprimen las comillas (en la primera edición de 1924 eran cursivas) con las que Rivera señaló lo que él consideraba provincialismos o regionalismos. [. . . ] Como ya la mayoría de esos términos han pasado a ser de uso corriente, mantener las comillas o las cursivas sólo haría más confusa la presentación del texto y más difícil la lectura de esta edición, que no pretende ser la edición crítica definitiva. Se aprovechó la intención cuestionadora de Rivera, sin embargo, para anotar y explicar precisamente estas palabras, a pesar de que aparecen en los diccionarios de la lengua (Ordóñez dans Rivera 1990 : 59).

Quant au glossaire final, son édition le reproduit avec fidélité, non sans une note qui signale ses limitations et ses inconséquences lexicographiques et sociolinguistiques. L'édition critique choisit ainsi de respecter la stratégie de Rivera, qui se présentait dans la fiction comme l'éditeur du manuscrit écrit par le protagoniste.

Faut-il rappeler que, dès leur première publication, chacun de ces trois romans s'est vu soumis à un long processus critique national et international qui, à partir de successives lectures idéologiques, a interrogé la validité esthétique et le degré de réalisme de leur hétérogénéité linguistique. Il a maintes fois été souligné que la confrontation entre le registre soutenu du langage savant et celui, populaire, du parler régional laissait transparaître l'ambivalence des auteurs à l'égard des cultures populaires régionales qu'ils prétendaient valoriser. Depuis la polémique argentine autour de l'image du gaucho dans Don Segundo Sombra, que les réalistes marxistes jugeaient par trop mythifiée, jusqu'aux lectures de La vorágine, qui mettent en relief l'image dégradée qu'offre le roman des Indiens d'Amazonie, en passant par l'interprétation manichéenne de Doña Bárbara à la lumière de la dichotomie entre civilisation et barbarie, les condamnations de ces tentatives, aussi sincères que contradictoires, de dialogisme romanesque n'ont pas manqué. 
Tranchant les controverses nationales autour de Don Segundo Sombra, Ernesto Sábato écrivait dans les années quatre-vingt :

Un crítico argentino, que pretende utilizar a Marx como maestro, sostiene que el Don Segundo Sombra de Güiraldes no existe, que es apenas la visión que un estanciero tiene del antiguo gaucho de la provincia de Buenos Aires; lo que es más o menos como acusar a Homero de falsificador porque exhaustivos registros llevados a cabo en las montañas calabresas y sicilianas no han dado con un solo cíclope. [. . . ] Que los sociólogos de la literatura ni los profesores de folklore no pierdan tiempo tratando de desautorizarlo (Sábato dans Güiraldes 1988 : XV).

Avec l'acuité et le talent qu'on lui connaît pour débattre de questions esthétiques concernant les usages d'un lexique local, Jorge Luis Borges avait déjà fait l'éloge du roman de Güiraldes dans sa très célèbre conférence de 1951 « El escritor argentino y la tradición », qui démolit les erreurs ou les fausses solutions du nationalisme littéraire. Borges sauve l'œuvre de Güiraldes de sa valorisation selon de seuls critères nationalistes en soulignant à quel point ce roman, ô combien, argentin est imprégné de littératures étrangères, tant dans son registre métaphorique proche des "métaphores des cénacles contemporains de Montmartre " ${ }^{2}$ (Borges 1989 [1953] : 271. Notre traduction), que dans son intrigue, inspirée du Kim de Kipling et de l'Huckleberry Finn de Twain. Il souligne ainsi les différences qui distinguent radicalement Don Segundo Sombra de la littérature gauchesca du XIXe siècle argentin. Aussi admirable qu'artificieuse, cette poésie gauchesca, explique Borges, prétend s'inspirer de la poésie populaire alors qu'elle en trahit l'ambition : traiter de sujets nobles dans un langage qui ne thésaurise pas de mots autochtones ni ne cherche à accentuer la couleur locale. Borges assène l'argument suivant pour souligner la différence entre le langage populaire affecté de la poésie gauchesca et le caractère authentiquement populaire de la poésie des gauchos : «La prueba es ésta: un colombiano, un mejicano o un español pueden comprender inmediatamente las poesías de los payadores, de los gauchos, y en cambio necesitan un glosario para comprender, siquiera aproximadamente, a Estanislao del Campo o Ascasubi. » (Borges 1989 [1953] : 268).

Le ferme rejet que préconise Borges face à la prolifération des localismes et à leurs corrélatifs glossaires censés garantir la nationalité littéraire est bien évidemment à entendre, non pas comme une condamnation sans appel de l'argentinité littéraire, mais comme un triple désir d'ouverture : à l'expression oblique de l'argentinité, insoupçonnée, tout aussi bien, de l'écrivain lui-même ; à une communauté de lecteurs constituée, d'emblée, par tous ceux qui lisent en espa-

2 Texte original : « metáforas de los cenáculos contemporáneos de Montmartre ». 
gnol ; à la littérature universelle. Et il est, bien entendu, assorti d'un éloge ou d'une défense de la traduction.

Le geste initial d'un Güiraldes, dont l'œuvre fait montre d'une tension féconde entre le local et l'universel - ce dernier étant fort modestement restreint à l'Europe et aux États-Unis -, répond néanmoins à un évident désir de précision lexicographique quant aux termes locaux de la province de Buenos Aires, où l'écrivain possède une estancia. Güiraldes collectionne amoureusement ces termes, tels des échantillons botaniques classés dans un herbier et travaille autant à partir de sa connaissance personnelle de ce lexique qu'avec des ouvrages spécialisés, dont il tente de pallier les éventuelles lacunes.

La preuve en est que la première édition, argentine et la seconde, espagnole, de son premier roman, Raucho (Buenos Aires, José Tragant, 1917 ; Madrid/Barcelone, Espasa Calpe, 1932), comprenaient un glossaire de localismes de la pampa, dont Güiraldes justifiait la nécessité avec l'indication suivante : « El siguiente vocabulario contiene palabras de uso común en nuestra provincia que no figuran en el Vocabulario Rioplatense Razonado, de Daniel Granada. » (Güiraldes 1932 : 259). Ce même glossaire a servi de modèle et de guide à ceux qui furent ajoutés aux éditions non argentines de Don Segundo Sombra. L'analyse génétique de ce dernier roman $^{3}$ souligne d'autre part que, durant le processus de correction du texte, l'écrivain prit soin de différencier le langage savant des passages narrés du parler que manient les personnages gauchos dans les dialogues et que, pour ce faire, il consultait le Pequeño Larousse Ilustrado (1913) de l'Espagnol Miguel de Toro y Gisbert pour décontaminer le registre savant de l'empreinte linguistique locale.

Un tel effort distinctif fait de l'auteur un sociolinguiste amateur, qui manie les localismes à bon escient et leur ménage une place dans la langue écrite sans pour autant s'écarter de la norme d'un espagnol général, sautant d'un registre à un autre comme un cavalier changerait de monture. Similaire, quoique sans doute moins agile, est le travail de Gallegos ou celui de Rivera à l'égard du langage des llaneros vénézuéliens et colombiens. Ni Gallegos ni Rivera n'étaient originaires des régions d'élevage de leurs pays respectifs comme l'était en revanche, du moins pour partie, Güiraldes, propriétaire terrien à San Antonio de Areco, dans la province de Buenos Aires. Sans doute l'urgence avec laquelle les deux écrivains introduisirent des glossaires dans les premières éditions de leurs romans respectifs est-elle due à cette moindre familiarité avec les parlers régionaux, à l'inexistence d'une tradition littéraire vénézuélienne ou colombienne comparable à la poésie gauchesca, à la publication en Espagne de Doña Bár-

3 Voir l'« Estudio filológico preliminar » de Élida Lois en Ricardo Güiraldes, Don Segundo Sombra, edición crítica de Paul Verdevoye, ALLCA, 1988, p. XLI-XLII. 
bara. De façon plus dynamique et plus subtile, une scène de La vorágine, qui narre l'initiation à leur nouvel environnement des amants de la capitale Alicia y Arturo Cova, transfère aux personnages de la fiction la jouissance qu'aura ressentie l'auteur en apprenant à manier le lexique des plaines : " Poco a poco el regocijo de nuestras lenguas fue cediendo al cansancio. Habíamos hecho copiosas preguntas que don Rafo atendía con autoridad de conocedor. Ya sabíamos lo que era una mata, un caño, un zural, y por fin Alicia conoció los venados » (Rivera 1990 [1924] : 92). C'est au partage de cette même jouissance esthétique et linguistique que sont conviés les lecteurs du roman. L'un des paris littéraires essentiels que renferme l'hétérogénéité linguistique desdits trois romans exemplaires de Rivera, Güiraldes et Gallegos consiste à éveiller chez le lecteur le plaisir de cette coexistence romanesque entre des registres et des régions de la langue, à lui apprendre à la lire, et pas seulement sur un plan lexical.

Imaginons un lecteur hispanophone des années trente, ni argentin de la pampa, ni colombien ni vénézuélien des plaines ou de la forêt vierge, un lecteur curieux qui, docile ou nécessiteux ou affligé de lexicomanie comme Baudelaire, consulterait et comparerait les glossaires initiaux ou ajoutés des trois romans. Certes, il s'émerveillerait de la diversité de ces intraduisibles termes locaux des univers de l'élevage ou de la récolte du caoutchouc mais il remarquerait bientôt la présence dans les trois glossaires de nombreux mots apparentés. La vorágine et Doña Bárbara partagent bien évidemment un même lexique des plaines d'élevage communes aux pays limitrophes que sont la Colombie et le Venezuela. Et si de l'un à l'autre roman, l'on trouve des variantes colombiennes et vénézuéliennes de ce lexique, celles-ci sont sans goute davantage dues à la sélection de termes qu'a faite chaque auteur qu'à une réelle différence terminologique. Mais même le glossaire de la pampa de certaines éditions de Don Segundo Sombra comprend certains mots qui, loin d'être locaux, appartiennent au lexique rural de l'art de l'élevage, qui est commun à nombre de parlers de l'espagnol. En voici quelques exemples pour le pur plaisir de la poétique que nous offre la ressemblance-dissemblance des acceptions et des variantes définitoires :

- Apadrinar: ayudar en la doma (DSS) / Amadrinador: jinete que acompaña al domador $(D B)$.

- Atropellada: embestida (DSS) / Atropellada: atropello (DB).

- Banco: extensión plana de terreno $(L V)$ / Banco: la parte prominente, de mayor o menor extensión, que sobresale de las sabanas $(D B)$.

- Barajustar: huir en tropel $(L V)$ / Barajustar: embestir, arremeter $(D B)$.

- Barajuste: dispersión, atropellada $(L V)$ / Barajuste: desbandada $(D B)$. 
- Boleadoras: instrumento de dos o tres bolas de piedra [. . .] forradas de cuero y sujetas a sendas guascas, que se arrojan a los pies o al pescuezo de los animales para aprehenderlos (DSS) / Bolerear: Hacer caer una bestia enlazándole las patas delanteras $(D B)$.

- Cabrestear: cuando la bestia sigue al que la lleva del cabestro (DSS) / Cabrestear (una punta de ganado): guiarla al esguazar un río, nadando delante de ella $(D B)^{4}$.

De glossaire à glossaire, de La vorágine à Doña Bárbara, se donnent ainsi la réplique les mots caribe, cazabe, coroto, corrido, chinchorro, maraca, morocota ou morrocota, puntero, topocho, trambucada, trambucarse.

On comprend aisément que Roberto Bolaño se soit amusé, lors de la réception du Prix Rómulo Gallegos à Caracas, à affirmer dans son discours que, dyslexique ou pataphysicien, il confondait les capitales de la Colombie et du Venezuela à cause du B/V de Bogotá et du Venezuela, et du C initial commun à Caracas et à la Colombie. En un iconoclaste bolivarisme littéraire, il jongla ensuite avec les titres de plusieurs romans du canon latino-américain, leur attribuant des nationalités, certes fantaisistes mais point injustifiées au regard de la logique de leurs stratégies littéraires. Les nationalismes ainsi moqués, il reste le territoire ou la région littéraire latino-américaine et, mieux encore, celui de la langue, l'Espagne y comprise. Bolaño conclut en disant être « chilien et aussi [. . . ] bien d'autres choses $»^{5}$ (Bolaño 2004 : 36) avant de déclarer que « la patrie d'un écrivain, c'est sa langue ", phrase qu'il reprend et dont il reconnaît le caractère quelque peu démagogique. Le corrélat en serait que la patrie d'un lecteur, c'est aussi sa langue, celle dans laquelle il lit, quand bien même il en lirait plusieurs. Et en ce sens, les romans régionalistes de Rivera, Güiraldes et Gallegos conduisent leur lecteur hispanophone vers leurs propres régions de la langue. Ils lui apprennent à apprécier le caractère forain de paysages lexicaux et idiomatiques qui, s'ils le déconcertent, deviennent siens grâce aux glossaires, à la façon dont tout locuteur est susceptible de s'approprier les nombreux termes du dictionnaire dont il n'use pas habituellement. Ce lecteur ressentira les localismes comme des « intraduisibles » qu'il pourra occasionnellement identifier au fil d'autres lectures, jouant

\footnotetext{
4 Les titres portent ici les abréviations suivantes : DSS pour Don Segundo Sombra, $L V$ pour $L a$ vorágine, $D B$ pour Doña Bárbara. Les glossaires respectifs consultés appartiennent aux éditions suivantes : Güiraldes, Ricardo (2000) : Don Segundo Sombra. Madrid : Jorge A. Mestas, Ediciones Escolares, « Clásicos universales », pp. 211-218 ; Rivera, José Eustasio (1926) : La vorágine. Bogotá : Editorial Minerva, tercera edición, pp. 313-314 ; Gallegos, Rómulo (1959) [1929] : Doña Bárbara, Obras completas, Tomo I. Madrid : Aguilar, pp. 800-803.
}

5 Texte original : « chileno y también [. . . ] muchas otras cosas ». 
avec la diversité terminologique des parlers régionaux de l'espagnol. Non moins bonne doit être la disposition du lecteur contemporain de littérature en espagnol qui ne cesse pas de (ne pas) traduire, sans avoir recours à des glossaires, des œuvres, point toujours emplies de localismes, des diverses traditions qui forment sa grande patrie.

Qui lira en traduction La vorágine, Don Segundo Sombra et Doña Bárbara se verra quelque peu frustré du plaisir, ou de la lassitude, si la disposition est mauvaise, que procure au lecteur hispanophone la diversité intralinguistique de l'espagnol. En effet, ce n'est pas un mince défi que de traduire ces romans. Les traduire avec une certaine justice impliquerait en l'occurrence un effort pour recréer le principe et l'effet de leur hétérogénéité linguistique malgré l'impossibilité manifeste de calquer le travail lexicographique des écrivains ou leur codification orthographique des parlers régionaux. Si les parlers régionaux de l'espagnol ne diffèrent pas autant entre eux que les dialectes de la péninsule italienne, qui se voient parfois confrontés à l'italien commun dans un même texte littéraire, la réflexion que propose l'italianiste, traducteur et poète JeanCharles Vegliante sur la traduction en français de tels exercices de diglossie littéraire peut nous orienter sur ce point. Dans un article de 1980, Vegliante montre, s'amusant à multiplier les traductions possibles d'un même fragment, à quel point il serait inapproprié de traduire dans tel ou tel parler régional ou tel ou tel argot du français les dialogues en dialecte triestin que manie le roman Ernesto (1953) du poète Umberto Saba. Vegliante rappelle la différence de statut entre les dialectes de l'italien et les variantes régionales du français, que l'histoire centralisatrice de cette langue a tendu à dénigrer. Et il souligne qu'il règne en Italie une situation de multilinguisme latent et plus ou moins conscient qui n'a pas d'équivalent en France. L'impossibilité de transposer en français la diglossie du roman de Saba lui inspire la conclusion suivante, dont il étend la portée à tout le champ de la traduction littéraire :

La traduction des dialectes, ainsi que la traduction tout court, dès qu'elle n'est pas simple annexion technique au sein d'une même réalité (désormais multinationale), ne saurait être transposition. Répétition créatrice, elle consiste à rechercher, dans et par le texte c'est-à-dire, évidemment, à inventer - un parcours singulier de subversion de la langue seul capable (de manière toujours provisoire, incomplète) de re-créer, de traduire, de « racheter dans sa propre langue ce pur langage exilé dans la langue étrangère » (W. Benjamin) (Vegliante 1980 : 82).

Comprenons que, sans relever de la diglossie au sens strict, l'hétérogénéité linguistique des romans de Rivera, Güiraldes et Gallegos rend plus patente encore que dans le cas d'œuvres plus homogènes l'aporie que représente la traduction comme simple transposition. C'est pourtant avec les limites que nous venons de voir et les stratégies de traduction qui régnaient dans la France des années 
trente et quarante du siècle dernier qu'ont relevé le défi les traducteurs en langue française de La vorágine, Don Segundo Sombra et Doña Bárbara.

Le cas de la traduction de Don Segundo Sombra, entreprise qui rassembla plusieurs traducteurs, des informateurs linguistiques et un correcteur, ne laisse pas d'être éclairant. Güiraldes, qui mourut en France un an après avoir achevé et publié son roman, avait eu le temps de prendre langue avec la traductrice Marcelle Auclair que lui avait présentée l'un de ses amis intimes : Valéry Larbaud. Pour apporter plus de soin encore à l'édition, le franco-uruguayen Jules Supervielle devait assister la traductrice en sa qualité de poète et de connaisseur bilingue de l'univers du Río de la Plata. Comme ce dernier l'indique dans sa préface à l'édition de Gallimard (1932), le travail avait été réparti entre trois personnes : Marcelle Auclair avait établi le sens du texte original en consultant, pour la compréhension du vocabulaire régional, tant le glossaire de Raucho que les œuvres des lexicographes Daniel Granada, Juan Valera et Miguel de Toro y Gisbert ; la traductrice avait ensuite élaboré la version française avec l'aide de son mari, l'écrivain Jean Prévost ; enfin, Supervielle avait révisé le tout. Saisi, semblerait-il, d'un prurit tout académique, le poète signale également que, dans la version traduite, les termes hippologiques proviennent du Cours d'Hippologie de Vallon. Et il n'omet pas de mentionner les contributions de la veuve et du frère de l'auteur pour l'éclaircissement $d u$ « vocabulaire argentin » non plus que les consultations effectuées auprès de l'écrivaine María Rosa Oliver. La préface de Supervielle semblerait ainsi vouloir décerner à la traduction un certificat de fidélité à l'original et garantir la propriété d'un lexique spécialisé, comme si le trio s'était efforcé de reproduire la quête d'authenticité de Güiraldes dans son évocation du parler et de la culture régionale de la pampa. Symptomatiquement, la description du processus de traduction met en relief trois étapes, dont la différenciation semblerait plus propre à la traduction de textes anciens ou spécialisés qu'à celle de romans contemporains : établissement du sens du texte original, version en français, révision et correction.

Tant de bonnes et de si scrupuleuses volontés auront évité à Marcelle $\mathrm{Au}$ clair des erreurs de compréhension du lexique régional ou hippologique et lui auront permis de déchiffrer la codification orthographique de la phonétique régionale. Le défi le plus élevé consistait néanmoins à recréer la grande, quoique subtile, distance que maintient le récit entre le registre soutenu de la narration à la première personne et le maniement de tournures orales ou encore l'effort pour mimer l'accent régional qu'arborent dans le roman les dialogues entre les gauchos ainsi que les contes de tradition orale que narre le personnage de Don Segundo. Et en effet, cette différenciation que Güiraldes avait tenu à polir et à repolir, n'apparaît pas avec une intensité similaire dans la version traduite : les indices orthographiques de l'accent régional disparurent fatalement, tandis que 
la présence de la syntaxe de l'oral se vit atténuée dans les dialogues. Quant à la narration des contes, les traducteurs firent appel aux conventions qui, dans la tradition française, codifient la transcription des récits de tradition orale, ce qui leur permit de se livrer à un exercice de transposition.

S’il est impossible d'apprécier, à la lecture de la version française quel fut l'apport de chaque membre du trio, on peut supposer que celui de Jean Prévost, en sa qualité d'écrivain exclusivement francophone, consista à éviter d'éventuels calques ou hispanismes afin de soigner l'expression finale en français. Rappelons qu'à l'époque prévalait encore dans la traduction littéraire et le monde de l'édition françaises sinon la tradition des « belles infidèles » du moins la tendance à opter pour la facile lisibilité de la version française, laquelle ne devait produire aucun effet d'étrangeté. Poussée à bout, la stratégie permettant d'atteindre ce but s'appelle, comme on sait, dans le jargon professionnel, " lisser le texte », le limer donc, lui ôter toute aspérité. L'exercice qui, pour les traducteurs de Don Segundo Sombra, consista à lisser la version française d'une œuvre originale dont le principal polissage tient à sa propre hétérogénéité linguistique, semble donc paradoxal sinon aporétique. À quels procédés pouvaient faire appel ces traducteurs pour opérer une recréation partielle en français de l'audace - car c'en était une à l'époque - de Güiraldes ? Maniant avec une extrême discrétion et autant de prudence les composantes linguistiques régionales et orales de l'original, ils se contentèrent de les évoquer au moyen d'indices en nombre restreint. Les traducteurs de Don Segundo Sombra auraient sans doute pu se montrer moins pusillanimes face au franc maniement de la syntaxe de l'oral dans les dialogues et seraient ainsi parvenus, en recréant l'écart entre le registre familier et le registre soutenu qui sous-tend le pari de Güiraldes, à une plus grande proximité du texte traduit avec l'original. Marcelle Auclair et Jean Prévost craignirent probablement, $d u$ fait de leur position ancillaire de traducteurs, que l'on ne juge négativement une telle expérimentation expressive. On ne manquait pourtant pas d'en lire de semblables dans certains romans français de l'époque, tel le Raboliot de Maurice Genevoix, qui remporte le Goncourt en 1925, se risquant à l'usage d'un parler rural de Sologne dans les dialogues que tiennent ses personnages. Se voyant hâtivement qualifié de régionaliste, le roman de Genevoix connut cependant une réception initiale qui ne rendait pas justice à sa portée poétique et à son registre symboliste. En matière d'audacieuse recréation littéraire de la langue orale française, dissociée en l'occurrence de tout régionalisme, Louis-Ferdinand Céline publie Voyage au bout de la nuit en cette même année 1932 qui voit la parution de la traduction française du roman de Güiraldes.

En sus du maniement parcimonieux, indiciel, de certain registre familier $\mathrm{du}$ français dans les dialogues, la discrète évocation de l'oralité régionale de Don 
Segundo Sombra consista à préserver dans la langue originale de rarissimes localismes ou argentinismes, assortis de notes explicatives en bas de page. L'on n'en trouve guère plus de cinq au total. Cas à part, une note signale que les traducteurs ont retenu le terme de " poney " pour désigner une espèce autochtone de chevaux («petisos »), fort différente des montures que l'on nomme ainsi en Europe. Une autre note propose la traduction de vers populaires cités en espagnol dans le corps du récit. Si les termes conservés dans la langue originale sont l'exception, la précision et la profusion des termes hippologiques ou de sellerie et de bourrellerie, peu usités et donc curieux dans la langue courante, placent le lecteur dans l'univers de l'élevage extensif, qui lui est, dans la plupart des cas, peu familier et qui participe d'un imaginaire lié au continent américain. De fait, le français avait d'ores et déjà lexicalisé quelques termes de l'espagnol comme lasso ou rodéo, qui connotaient indifféremment tout l'élevage des vastes terres américaines. Ainsi, une fois le lexique régional de la pampa fondu dans un français courant, le lexique spécialisé - hippologique, sellier ou d'élevage - pallie l'absence de localismes, apportant au lecteur français un certain effet d'estrangement. Déjà initié dans les années trente au monde américain d'« Élevage " par les romans de Fenimore Cooper, les récits de voyage en Argentine de quelques auteurs français ou les romans d'aventures français - désormais fort oubliés - dont l'action se déroule dans le Mexique d'après l'Intervention française, ce lecteur aura identifié dans l'univers de Don Segundo Sombra l'une des régions de ce monde lointain : la pampa du Sud du continent. Encore heureux s'il la distingue clairement des autres et qu'il comprend l'unité si diverse de ce monde que j'appelle ici «Élevage » en m'inspirant de l'usage toponymique, générique et poétique que fait Édouard Glissant du mot « Plantation ». Rappelons que le poète et essayiste martiniquais baptise ainsi la diffuse région américaine où, du Sud faulknérien des États-Unis jusqu'au Brésil, en passant par les Antilles, se répète une même structure économique, sociale et culturelle fondée sur l'esclavage. Si, en Argentine, Güiraldes modernise la tradition de la littérature gauchesca en écrivant un roman de formation aussi poétique qu'anthropologique, les traducteurs de la version française de Don Segundo Sombra évitèrent, sans l'avoir peut-être calculé, le risque que l'œuvre se prête à une lecture par trop exotiste, en restreignant au maximum la reprise des localismes originaux assortis de leurs corrélatives notes de bas de page. Cette stratégie permet paradoxalement de mieux apprécier l'autochtonie, l'originalité et la qualité littéraire de l'œuvre de Güiraldes, en la distinguant radicalement de tout type d'œuvres se complaisant dans l'exotisme.

Confirmant la réflexion de Borges quant à l'espagnol courant qui caractérise l'authentique poésie des gauchos, les vers chantés ou les couplets dansés de la pampa que recueille le chapitre XI du roman font montre de très rares lo- 
calismes. Perçus comme hautement traduisibles du fait qu'ils appartiennent, à l'image des contes de tradition orale, à un patrimoine des formes populaires censément universel - et de fait très européen -, vers et couplets furent adaptés aux conventions de formes jugées équivalentes dans la tradition littéraire française, telles les ballades ou les chansons populaires. Sans doute doit-on au poète Supervielle qu'en hommage à la création populaire le premier distique soit cité dans la langue originale et traduit dans une note de bas de page. Georges Pillement et René L. F. Durand, les traducteurs respectifs de La vorágine et de Doña Bárbara, purent notoirement avoir recours au même type de transposition pour traduire les vers populaires qu'improvisent les personnages de ces romans dans des scènes de veillées des plaines. Tout comme la traduction française de Don Segundo Sombra, celle que fit Pillement de La vorágine (La Voragine, Bellenand, 1951) cite en espagnol les deux premières strophes versifiées qui apparaissent dans le roman, n'en proposant une version française en note que dans le cas de la deuxième, avant de traduire les suivantes dans le plein texte. Dans sa traduction de Doña Bárbara (Dona Barbara, Gallimard, 1951) René L. F. Durand, ne cite pas, en revanche, les strophes des chansons populaires dans leur version originale. Qu'il s'agisse de rendre hommage à la poésie populaire ou du souhait de produire un effet d'authenticité référentielle, le fait de citer initialement les vers en espagnol ou de les proposer en version bilingue installe dans les traductions une hétérogénéité linguistique qui récrée autrement le geste anthropologique des romanciers. Dans les œuvres originales, la présentation typographique de ces vers, en italiques et dans des paragraphes isolés, les met en relief à la manière de citations, ce qui, facilitant leur appréhension comme textes autonomes et allographes, semblerait favoriser leur transcription directe dans les versions traduites des romans.

Si les traducteurs de Don Segundo Sombra s'en tinrent au principe de la plus grande restriction dans l'usage des localismes et des notes, tant Georges Pillement que René L.-F. Durand, confrontés, pour leur part, aux glossaires de La vorágine et de Doña Bárbara, eurent recours avec plus ou moins de bonheur au maniement partiel des localismes originaux assortis d'un abondant appareil de notes définitoires : 64 dans le cas de La Voragine, 39 dans celui de Dona Barbara. Le patrimoine lexical que proposaient en annexe José Eustasio Rivera et Rómulo Gallegos fut donc réparti tout au long des deux romans. Au fur et à mesure que progressent les textes et que vont se définissant les termes locaux signalés par des italiques, la fréquence des notes diminue et la lecture s'allège pour qui aura, suppose-t-on, mémorisé le sens de tel ou tel mot. La traduction que fit René L.-F. Durand de Doña Bárbara se distingue néanmoins de celle de Pillement par la précision de ses notes explicatives et par la recherche inspirée de termes usuels dans les Antilles françaises pour traduire des localismes vénézuéliens qui désignent des objets ou des types de métissage similaires : ainsi « caney» 
est-il traduit par « carbet » et « catire », par « chabin », non sans note définitoire. Quoi qu'il en soit, ces traductions revêtent un aspect quasi philologique qui, tout en se guidant sur le travail lexicographique qu'avaient déployé Rivera ou Gallegos, arbore nécessairement sur le plan lexical et phonétique une hétérogénéité linguistique bien plus marquée que celle des œuvres originales.

$\mathrm{Si}$, dans ces dernières, les localismes étaient maniés comme des « intraduisibles ", leur simple importation dans les traductions françaises en fait des « intraduisibles » au carré et peut induire une lecture des romans qui tende à être plus exotiste qu'anthropologique. En ce sens, la traduction de Don Segundo Sombra, avare de localismes, semblerait plus contemporaine, bien qu'elle soit parue en 1932, que celles de La vorágine ou Doña Bárbara, publiées toutes deux en 1951. La preuve en est que les éditions Phébus l'ont republiée en 1994, avec un commentaire élogieux de l'éditeur Jean-Pierre Sicre, qui la juge « exemplaire » (Güiraldes 1994 : 11). Et elle paraît plus contemporaine car les actuelles politiques éditoriales du monde global privilégient les stratégies de traduction qui facilitent un confort de lecture, avec une corrélative chasse aux notes du traducteur, réservées aux éditions érudites. Il n'y a pas forcément lieu d'y voir un retour à la tradition du texte lissé de ses étrangetés ou de ses aspérités dans sa version traduite. En effet, on prête au public lecteur contemporain, sans doute plus conscient de la diversité des langues que celui du siècle dernier, éventuellement plus polyglotte, une certaine capacité à se livrer à une lecture hétérolingue, un certain consentement face à l'étrange, le forain ou l'étranger. Par exemple : après les romans de Patrick Chamoiseau et de Raphaël Confiant ou les essais d'Edouard Glissant, le mot antillais « chabin » exigerait-il une note de bas de page pour la seule raison qu'il ne se trouve pas dans le Larousse?

Dans le paysage littéraire et artistique contemporain, où les œuvres traduites conservent bien souvent les titres des œuvres originales ${ }^{6}$, où les récits offrent de timides amorces ou tentatives d'hétérolinguisme, on ne franchit toujours pas impunément les frontières linguistiques. Mais peut-être les lecteurs que nous sommes campons-nous avec moins de frayeur dans les zones franches ou sauvages de l'intraduisible, peut-être les traducteurs que nous sommes pouvons-nous en jouir avec une humble audace, prêts à tailler des brèches dans la broussaille ou la forêt, au risque qu'elles se referment sur notre passage, certains que d'autres viendront, qui en ouvriront de nouvelles.

6 Soulignons que dans les années trente ou cinquante, les titres de nos trois romans exemplaires avaient à peine été lexicalisés en français. 


\section{Bibliographie}

Bolaño, Roberto (2004) : «Discurso de Caracas », Entre paréntesis. Barcelona : Anagrama.

Borges, Jorge Luis (1989) : «El escritor argentino y la tradición ». In : Obras completas 1923-1949. Barcelona: Emecé, pp. 267-274.

Cassin, Barbara (ed.) (2004) : Vocabulaire européen des philosophies. Dictionnaire des intraduisibles. Paris : Seuil/Le Robert, "Sciences humaines ».

- (2016) : Éloge de la traduction. Compliquer l'universel. Paris : Fayard, «Ouvertures». Gallegos, Rómulo (1959 [1929]) : Doña Bárbara, Obras completas, Tomo I. Madrid : Aguilar.

- (1997 [1929]) : Doña Bárbara, edición crítica de Domingo Miliani. Madrid : Cátedra, « Letras hispánicas ».

- (1951 [1926]) : Dona Barbara, trad. René L.-F. Durand. Paris : Gallimard, « La Croix du Sud ».

Güiraldes, Ricardo (1966 [1929]) : Don Segundo Sombra. Buenos Aires : Losada, vigésima tercera edición.

- (1988 [1926]) : Don Segundo Sombra, edición crítica de Paul Verdevoye. Paris : « Archivos » ALLCA-Unesco.

- (2000 [1926]) : Don Segundo Sombra. Madrid : Jorge A. Mestas, Ediciones Escolares, "Clásicos universales ".

- (1953 [1932]) : Don Segundo Sombra, trad. de Marcelle Auclair, revue par Jean Prévost et Jules Supervielle, préface de Jules Supervielle. Paris : Gallimard, "La Croix du Sud », 11ème édition.

- (1994) : Don Segundo Sombra, trad. de Marcelle Auclair, revue par Jean Prévost et Jules Supervielle. Paris : Phébus.

Rivera, José Eustasio (1926 [1924]) : La vorágine. Bogotá : Editorial Minerva, tercera edición.

- (1990 [1924]) : La vorágine, edición crítica de Montserrat Ordoñez. Madrid : Cátedra, "Letras hispánicas".

- (1951) : La Voragine, trad. de Georges Pillement. Paris : Éditions Bellenand.

Vegliante, Jean-Charles (1980) : «Traduction, traduction des dialectes, interlangue ». In : Les langues modernes LXXIV, 1, pp.78-84. 
